Proc. XIX International School of Semiconducting Compounds, Jaszowiec 1990

\title{
OPTICS OF ZERO DIMENSIONAL SEMICONDUCTOR SYSTEMS
}

\author{
A.I. Ekrmov and AL.L. EFros \\ S.I. Vavilov State Optical Institute, 199164 Leningrad, USSR
}

(Received August 8, 1990)

\begin{abstract}
The optical spectra of semiconductor microcrystals grown in transparent matrix of oxide glass are investigated. The size of microcrystals was varied in a controlled manner from a few tens to a few hundreds of angstroms. The microcrystal embedded in wide gap matrix represents three-dimensional potential well for electrons, holes and excitons. The optical properties of such zero-dimensional semiconductor structures are shown to be governed by the structure of energy spectra of confined electron-hole pairs. The phenomenon of the microcrystals ionization at interband optical excitation is observed. The Auger process in microcrystals containing two nonequilibrium electron-hole pairs is proposed to be responsible for this effect. The experimental dependencies of the ionization rate as a function of excitation intensity and the microcrystal size are in a good agreement with the theoretical predictions of the Auger recombiantion model.
\end{abstract}

PACS numbers: $78.90 .+t$

Optics of semiconductor microcrystals embedded in dielectric matrices have been a subject of very intensive investigations for the last years $[1,2]$. Actually, there are two reasons for this. The first reason is a fundamental character of phenomena which dominate the energy spectrum of electronic states in microcrystals. It is well established now that a semiconductor microcrystal in a wide-band matrix represents a three-dimensionally confined quantum well for quasiparticles. When microcrystals have sizes comparable to the Bohr radius of the exciton in the bulk semiconductor the resulting quantum confinement strongly modifies the optical spectra. The second reason is possible device applications which are due to the expected largely enhanced optical nonlinearities [3] and electro-optical effects [4] in semiconductor-doped glasses.

The purpose of this talk is to give a short review of the present situation in the studies of the zero-dimensional semiconductor systems. The growth technique and optical spectra of microcrystals in a glass matrix will be discussed at some length. Another problem to be discussed is the energy spectra of zero-dimensionally 
confined electron-hole pairs in quantum semiconductor dots. And finally, the role of Auger-processes in photoionization of semiconductor microcrystals in a glass matrix will be regarded.

\section{Semiconductor microcrystals in a glass matrix: growth and optical spectra}

The developed technologies make it possible to grow the microcrystals of semiconductor compounds in glassy [5], crystalline [6] and aqueous matrices [7]. The growth of microcrystals in solid matrices is based on the process of diffusion - controlled phase decomposition of a super saturated solid solution. The recondensation stage of the process, when the large particles grow at the expense of dissolution of small ones, was investigated theoretically by Lifshits and Slesov. The kinetics of growth is described by the following expression [8]

$$
\bar{a}=\left(\frac{4}{9} \sigma C D \tau\right)^{\frac{1}{3}}
$$

where the diffusion coefficient $D$ and equilibrium concentration of the solution $C$ depend exponentially on temperature; $\sigma$ is a coefficient proportional to interfacing surface tension, $\tau$ - time duration of annealing process. Mean value of the microcrystal radius may be measured off small-angle X-ray scattering [5] or with the use of TEM technique [9]. It was also shown that a steady-state size distribution is formed in the course of recondensation growth and an analytical expression for it was obtained [8].

Thus, the technique of diffused-controlled growth of semiconductor microcrystals makes it possible to vary the size of particles starting from a few tenths of angstroms. The steady-state size distribution of microcrystals is rather narrow and may be taken into account in calculations, since the analytical expression for it is known. Further investigations of the growth process are in progress now. A biosynthesis of CdS microcrystals was announced recently [10]. Sol-gel technology of silicate glass production was shown to be useful for microcrystals growth [11]. The Ge microcrystals embedded in $\mathrm{SiO}_{2}$ thin films were obtained with rf sputtering technique [12].

The optical transparency of an oxide glass matrix makes it possible to apply all of the methods of optical spectroscopy to study such zero-dimensional system. The Figure 1 shows the absorption spectra of glass samples containing CdSe, CdS, $\mathrm{CuBr}$ and $\mathrm{CuCl}$ microcrystals. It is seen that spectra reveal the typical excitonic structure of near band-gap transitions. This structure is due to the spin-orbit splitting in cubic materials $(\mathrm{CuBr}, \mathrm{CuCl})$ and to the spin-orbit and crystal field splitting in hexagonal semiconductors (CdS, CdSe). The absorption spectra as well as luminescence and Raman spectra show that the semiconductor particles grown in glass matrix have the crystalline structure and sufficiently high spectroscopic quality. The X-ray scattering experiments were carried out recently for investigation of structural modifications of $\mathrm{CdS}[10]$ and $\mathrm{CdSe}[13]$ microcrystals. 


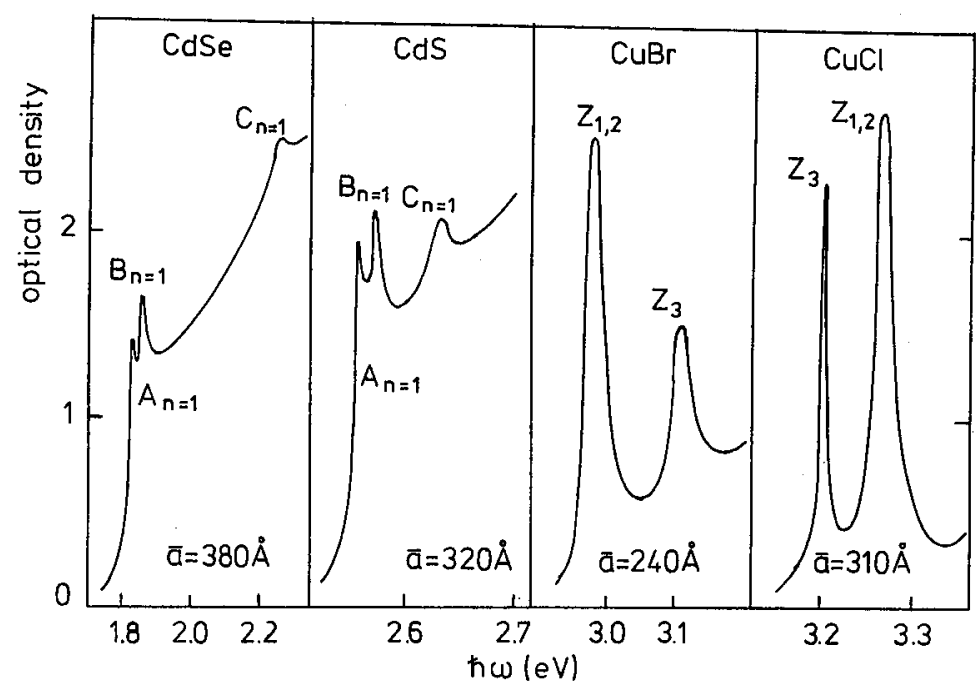

Fig.1. Low temperature $(T=4.2 \mathrm{~K})$ absorption spectra of glasses with microcrystals: $\operatorname{CdSe}(\bar{a}=380 \AA), \operatorname{CdS}(\bar{a}=320 \AA), \operatorname{CuBr}(\bar{a}=240 \AA)$, and $\operatorname{CuCl}(\bar{a}=310 \AA)$.

\section{Energy spectrum of electronic states in semiconductor quantum dots}

The semiconductor-doped glasses are of interest as a new class of objects which may be used for the investigation of quantum confined effects in semiconductors. Besides size quantization, the spectra of electron-hole pairs are also influenced by the Coulomb interaction, the energy of which depends on microcrystal size too. So, the different cases, depending on relationship between these two energies have to be regarded.

The Figure 2a shows the absorption spectra of glasses doped with $\mathrm{CuCl}$ microcrystals of different sizes. As can be seen, the absorption is of excitonic nature down to smallest sizes. The decrease of the radius of microcrystals leads to high blue shift for both excitonic lines. This effect is due to the size quantization of an exciton as a whole, because the binding energy of the exciton in the material is very $\operatorname{high}\left(E_{e x} \approx 0.2 \mathrm{eV}\right)$ and the radius of exciton is rather small $\left(r_{e x} \approx 8 \AA\right)$. The size dependence of spectral position of both excitonic lines are shown in Fig. 2b. For the exciton originating from the upper, nondegenerate subband $\Gamma_{7}$ the dependence is described by simple expression

$$
\hbar \omega_{Z_{3}}=E_{g}-E_{e x}+0.67 \frac{\hbar^{2}}{2 M_{s} \bar{a}^{2}} \pi^{2}
$$

where $E_{g}$ is the band gap and $E_{e x}$ is the exciton binding energy. The numerical factor results from the averaging of the Lifshits size distribution function. The slope of the curve is determined by the value of the excitonic translation mass $M_{s}$. 

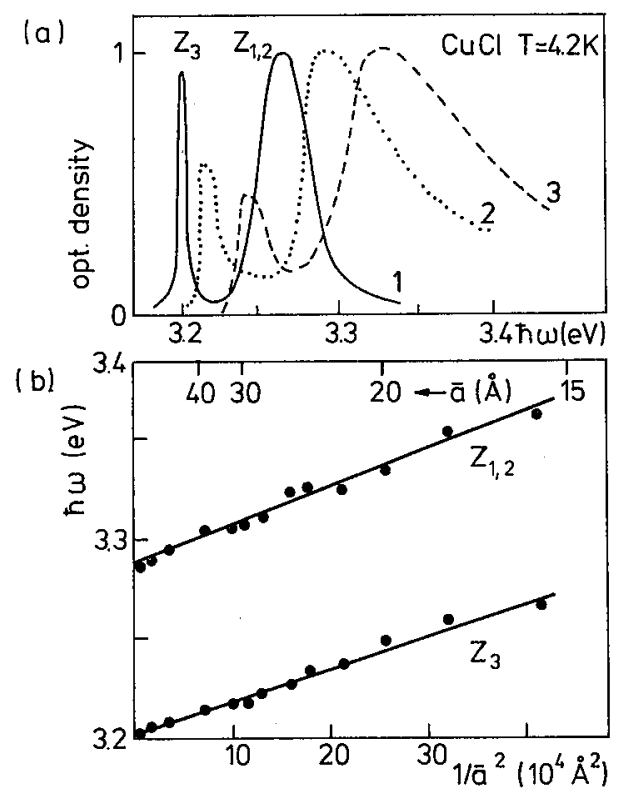

Fig.2. (a) Absorption spectra of glasses with CuCl microcrystals: (1) $\bar{a}=310 \AA$, (2) $\bar{a}=$ $29 \AA,(3) \bar{a}=20 \AA$. (b) Spectral position of oscillations vs. $1 / \bar{a}^{2}$.
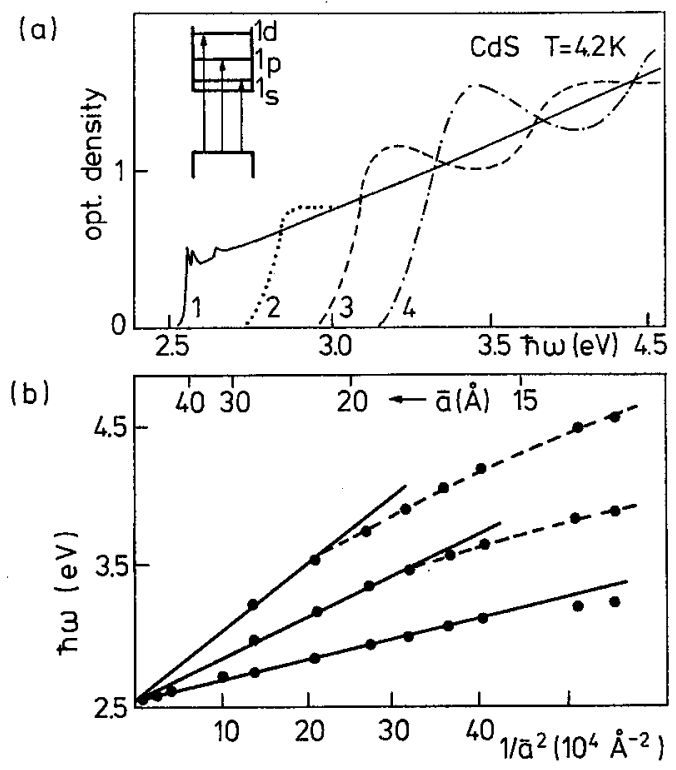

Fig.3. (a) Absorption spectra of glasses with CdS microcrystals: (1) $\bar{a}=330 \AA$, (2) $\bar{a}=$ $23 \AA$, (3) $\bar{a}=15 \AA$, (4) $\bar{a}=12 \AA$. (b) Spectral positions of oscillations vs. $1 / \bar{a}^{2}$. 
The special treatment is needed to describe the size dependence of spectral position for excitons originating from the degenerate valence subband $\Gamma_{8}$. It was shown that this dependence is given by the following expression [14]

$$
\hbar \omega_{Z_{1,2}}=E_{g}-E_{e x}+\Delta+0.67 \frac{\hbar^{2}}{2 M_{h} \bar{a}^{2}}\left[\Phi\left(M_{l} / M_{h}\right)\right]^{2}
$$

where $M_{h}$ and $M_{l}$ are the "heavy" and "light" exciton masses, $\Delta$ is the value of spin-orbit splitting, $\Phi\left(M_{l} / M_{h}\right)$ is the root of transcendental equation [14].

Comparison between experimental and theoretical results enables us to determine the masses of exciton for all three exciton subbands: $M_{s}=(1.9 \pm 0.2) m_{0}$, $M_{h}=(2.6 \pm 0.2) m_{0}$, and $M_{l}=(1.5 \pm 0.2) m_{0}$. It is necessary to emphasize that the effective mass approximation gives the possibility to describe experimental results for the values of microcrystal radius down to about $\bar{a} \approx 15 \AA$.

The absorption spectra of CdS microcrystals of different sizes are shown in Fig. 3a. It is seen that for the semiconductor material with a low binding energy of the exciton $\left(E_{e x} \approx 30 \mathrm{meV}\right.$ for $\mathrm{CdS}$ ) the decrease of microcrystal size leads to a large blue shift of the absorption band edge. Oscillations in absorption spectra of microcrystals with radius less than the exciton radius $\left(r_{e x} \approx 30 \AA\right)$ are due to quantum sublevels of conduction band [15]. As it is seen in Fig. 3b, the simple analytical expression

$$
\hbar \omega_{l, n}=E_{g}+0.71 \frac{\hbar^{2}}{2 m \bar{a}^{2}} \Phi_{l, n}^{2}
$$

with the electron effective mass $m_{e}=0.2 m_{0}$ gives the possibility to describe the size dependence of the blue shift only for the forbidden gap width. It is clear that a nonparabolicity of the electron dispersion law and the finite depth of the quantum well have to be taken into account. The theoretical investigations of the energy spectrum of electron-hole pairs for the real band structure of the material under investigation are in progress now [16].

\section{Auger-ionization of semiconductor microcrystals in glass matrix}

It is well known now that optical properties of semiconductor-doped glasses are affected by photon fluency. One may observe light-induced effects of the luminescence intensity degradation (the darkening effect [3]), changes of microcrystals optical absorption spectra [17] and a decrease of the carrier life-time [18]. All of the effects are usually revealed at room temperatures but heating at $300-400^{\circ} \mathrm{C}$ results in their disappearance.

We suggest that these effects are due to the photoionization of microcrystals in a glass matrix. The possibility of that process was demonstrated experimentally in the studies of the thermally stimulated luminescence (TSL) [17]. Figure 4a shows the TSL curves for two samples which have been illuminated at $T=77 \mathrm{~K}$ with light and then heated at a constant rate $0.1 \mathrm{~K} / \mathrm{s}$. The first sample was undoped and the second one was doped by CdS microcrystals with $\bar{a} \approx 30 \AA$. The first sample was subjected to UV-light irradiation with $\hbar \omega_{e x} \approx 6 \mathrm{eV}$ which is in the spectral range of the interband absorption of the glass. The TSL curve for this sample 


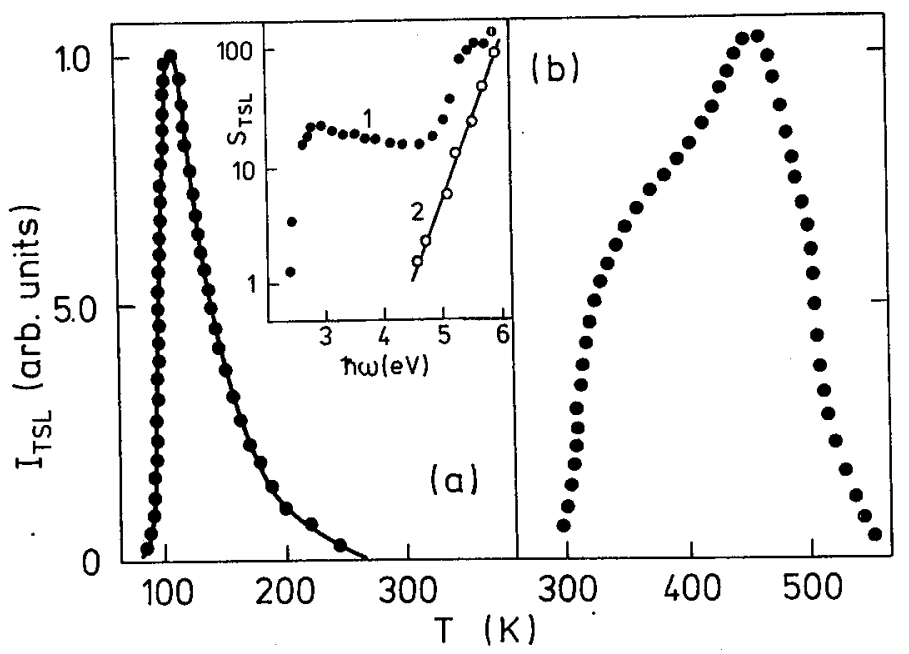

Fig. 4. Thermally stimulated luminescence curves of undoped (-) and CdS-doped glass $(\cdots \cdots)$. (a) $T_{e x c}=80 \mathrm{~K}$; (b) $T_{\text {exc }}=300 \mathrm{~K}$. Inset shows the spectral dependence of the efficiency of ionization $S_{\text {TSL }}$ for (1) undoped glass and (2) CdS-doped glass.

which was measured within the spectral range of the intrinsic luminescence of glass is shown in Fig. 4a by a solid line. It is a typical curve which is due to the well-known electron capture centers in the glass ( $E_{1}$-centers [19]).

The second sample doped with CdS microcrystals was subjected to light irradiation from the region of the absorption edge of the microcrystals $(\hbar \omega \approx 3.5 \mathrm{eV})$. The TSL in this sample was registered within the spectral range corresponding to the impurity luminescence of the microcrystals. This curve is shown in Fig. 4a by points. A coincidence of these curves directly shows that nonequilibrium electrons have a finite probability to leave microcrystals and be captured by the same capture centers of glass in the vicinity of the microcrystal. Sample heating leads to going back of the electrons to microcrystals with their subsequent radiative recombination with holes. The inset in Figure 4a shows the spectral dependence of the ionization efficiency for microcrystals. It is seen that the ionization starts at the energies of the excitation quanta corresponding to the band gap of microcrystals. The increase of the efficiency at the energy of about $4.5 \mathrm{eV}$ is due to the above-barrier transitions of electrons from the microcrystal into the glass.

The effect of the photoionization of microcrystals under photon flux is also observed at room temperature. Figure $4 \mathrm{~b}$ shows the relevant TSL curve. It is seen that electrons in this case are captured by deeper traps and heating to about $600 \mathrm{~K}$ is needed to empty them.

We suppose that the process of the photoionization of microcrystals is accompanied by a decrease of the carrier life-time, degradation of the luminescence intensity, and other light-induced effects due to the strong enhancement of nonradiative processes in them. The mechanism of the nonradiative recombination in 


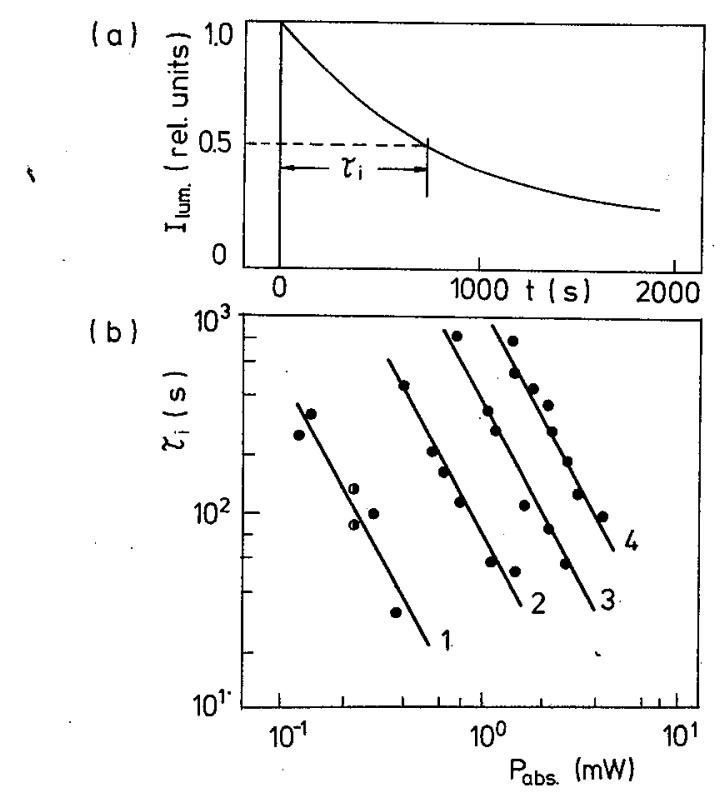

Fig.5. (a) The dependence of luminescence intensity on time of steady state excitation for CdS microcrystals with $\bar{a}=22 \AA$. (b) The dependence of ionization time $r_{i}$ on absorbed power for microcrystals with different sizes (1) $\bar{a}=13 \AA$, (2) $16 \AA$, (3) $22 \AA$, (4) $33 \AA$.

the ionized microcrystal is beyond the scope of today's discussion. We shall discuss now the microscopic mechanism of the photoionization of microcrystals.

Figure 5a shows the dependence of the photoluminescence intensity on time of optical excitation measured on the samples with $\bar{a}=22 \AA$. The $\lambda=406$ $\mathrm{nm}$ line of a krypton laser was used for the steady-state excitation. Studies were performed at the temperature of $T=77 \mathrm{~K}$. The kinetics of the degradation is not exponential and, to a first approximation, we shall characterize it by the time $\tau_{i}$ during which the intensity is diminished by a factor of 2 . Figure $5 \mathrm{~b}$ shows the degradation kinetics (in logarithmic scale) as a function of the absorbed power for the samples of different microcrystals sizes. The ionization time $\tau_{i}$ is seen to be strongly size-dependent and also inversely proportional to the square of the absorbed power. This observation indicates the two-photon character of the ionization process.

The qualitative model used for the analysis of the experimental results is as follows:

1. The ionization of microcrystals is the result of the Auger recombination process in the microcrystals containing two nonequilibrium electron-hole pairs. It is the many-electron process in which the energy of the electron-hole pair annihilation is transferred to the electron. If this energy is larger than the 
barrier height, the electron ejects into glass and the ionization of microcrystals takes place. It is worthy to mention here that the role of the Auger processes in microcrystals at high excitation densities has been demonstrated experimentally [3].

2. The ionized microcrystals have a very low quantum efficiency of luminescence. A strong enhancement of the nonradiative channel may, for instance, be the result of an effective Auger recombination in ionized microcrystals since three quasiparticles (one electron and two holes) appear as a result of the one-photon excitation.

Thus, the luminescence intensity reflects in this case the number of yet nonionized microcrystals. The ionization rate $\left(1 / \tau_{i}\right)$ is equal to the probability for the existence of the microcrystals with two nonequilibrium electron-hole pairs times the Auger annihilation rate

$$
\tau_{i}^{-1}=\left(W_{01} \tau_{0}^{1}\right)\left(W_{02} \tau_{0}^{2}\right) \tau_{A}^{-1}
$$

where $W_{01}$ and $W_{02}$ are the probabilities of photon absorption in nonexcited microcrystals and microcrystals containing one electron-hole pair; $\tau_{0}^{1}$ and $\imath_{0}^{2}$ are carrier life-times in the microcrystals with one and two electron-hole pairs. $\tau_{A}^{-1}$ is the probability of the Auger recombination in the microcrystal with two electron-hole pairs. At low intensity of excitation $W_{01}$ and $W_{02} \sim I_{e x}$.

The calculation of the Auger recombination probability is a difficult problem because it demands the exact calculations of the small overlap integrals of the electron-hole states. Therefore, the electron-hole wave functions of the zero-dimensional quantum size structures should be considered within the many--band approximation. The second problem is that the electron ejection from the microcrystal (ionization of them) demands consideration of the quantum structure with the finite depth of the quantum well. This consideration was made within the framework of the Kane model by Al. Efros and V. Kharchenko [16]. Size dependence of the rate of the Auger ionization in microcrystals was obtained by numerical calculations for CdS $\left(E_{g}=2.6 \mathrm{eV}, m_{e}=0.2 m_{0}\right)$ microcrystals embedded in the matrix of an oxide glass $\left(E_{g}=7 \mathrm{eV}\right)$. The dependence obtained is shown in Fig. 6 by the solid line. The probability of the Auger ionization is seen to be of a pronounced oscillatory character. The maxima are related to the microcrystals where electronic quantum-size levels cross the boundary of a continuous spectrum or are situated in the neighborhood of it. In real glass samples the averaging of oscillations of $1 / \tau_{A}$ takes place due to dispersion of microcrystal sizes and shapes, fluctuation of the microcrystal-glass band offset, etc.

We obtained the experimental values of $\tau_{A}(a)$ from experimental dependence $\tau_{i}(\bar{a})$ using Eq.5. For that it is necessary to estimate the steady state share of microcrystals containing two electron-hole pairs. Estimating that one as the square of steady state share of one excited microcrystal $W_{01} \tau_{0} \approx 10^{-5}$ we obtain in our experiment that $\tau_{A}=\left(W_{01} \tau_{0}^{1}\right)^{2} \tau_{i} \approx 10^{-10} \tau_{i}$ (the value $W_{01} \tau_{0}^{1} \approx 10^{-5}$ was estimated from the absorbed power). The probability of Auger-disintegration $\tau_{A}^{-1}$ 


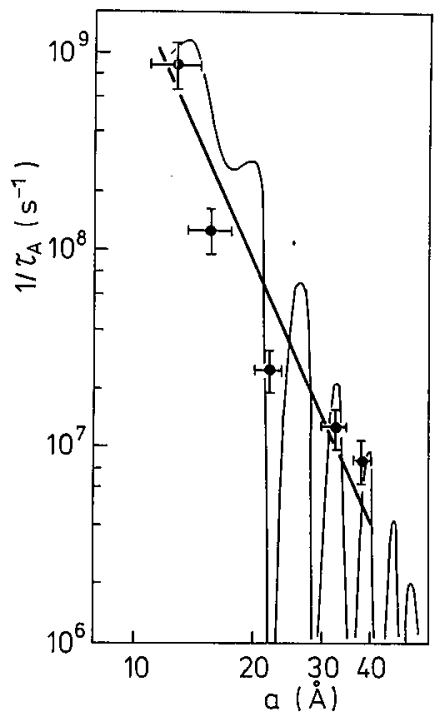

Fig.6. The theoretical dependence of the Auger ionization probability of CdS microcrystals on their radius. The crosses show the experimental data.

obtained in such a way is shown in Fig. 6 by crosses. It is seen that the experimental data are in a good agreement with theoretical dependence.

Thus the obtained results demonstrate that the ionization of microcrystals embedded in the glass matrix is due to the Auger recombination which takes place in the microcrystals containing two photoexcited electron-hole pairs at the same time. Further studies, both experimental and theoretical, have to be performed to understand the energy spectrum of the ionized microcrystals and features of relaxation and recombination processed in them.

\section{References}

[1] A.I. Ekimov, Al.L. Efros, A.A. Onushchenko, Solid State Commun. 56, 921 (1985).

[2] D.S. Chemla, D.A.B. Miller, J. Opt. Soc. Am. B 2, 1155 (1985).

[3] P. Roussignol, D. Ricard, J. Lukasik, C. Flytzanis, J. Opt. Soc. Am. $B$ 4, 5 (1987).

[4] A.I. Ekimov, A.P. Scvortsov, T.V. Shubina, S.K. Shumilov, Al.L. Efros, J. Tech. Phys. 59, 202 (1989).

[5] V.V. Golubkov, A.I. Ekimov, A.A. Onushchenko, V.A. Tsekhomskii, Fiz. Khim. Stekla 7, 397 (1981).

[6] T. Itoh, T. Kirihara, J. Lumin. 31, 120 (1984).

[7] R. Rossetti, S. Nakahara, L.E. Brus, J. Chem. Phys. 79, 1086 (1983). 
[8] I.M. Lifshits, V.V. Slesov, Zh. Eksp. Teor. Fiz. 35, 479 (1958).

[9] N.F. Borrelli, D.W. Hall, H.J. Holland, D.W. Smith, J. Appl. Phys. 61, 5399 (1987).

[10] C.T. Dameron, R.N. Reese, R.K. Mehra et al., Nature 338, 596 (1989).

[11] S. Modes, R. Lianos, Chem. Phys. Lett. 153, 351 (1988).

[12] S. Hayashi, M. Fujii, K. Yamamoto, Jpn. J. Appl. Phys. 28, L1464 (1989).

[13] M.G. Bowendi, A.R. Kortan, M.L. Steigerwald, L.E. Brus, J. Chem. Phys. 91, 7282 (1989).

[14] A.I. Ekimov, A.A. Onushchenko, A.G. Plukhin, Al.L. Efros, Sov. Phys.- JETP 61, 891 (1985).

[15] A.I. Ekimov, A.A. Onushenko, JETP Lett. 40, 1136 (1984).

[16] Al.L. Efros, V.A. Kharchenko, M.G. Ivanov, in Proc. of Allunion Conf. Theory of Semiconductors, Doneck 1989, p. 36.

[17] V.V. Grabovskis, Y.Y. Dzenis, A.I. Ekimov et al. Sov. Phys. - Solid State 31, 149 (1989).

[18] J.P. Zheng, H.S. Kwok, Appl. Phys. Lett. 54, 1 (1989).

[19] J.H. Mackey, H.L. Smith, A.I. Halperin, J. Phys. Chem. Solids 27, 1759 (1966). 